3 Wilkes MR, Sereika SM, Fertig N, Lucas MR, Oddis CV. Treatment of antisynthetase-associated interstitial lung disease with tacrolimus. Arthritis Rheum 2005; 52: 2439-2446.

4 Sauty A, Rochat T, Schoch OD, et al. Pulmonary fibrosis with predominant CD8 lymphocytic alveolitis and anti-Jo-1 antibodies. Eur Respir J 1997; 10: 2907-2912.
5 Soccal PM, Gasche Y, Favre H, Spiliopoulos A, Nicod LP. Improvement of drug-induced chronic renal failure in lung transplantation. Transplantation 1999; 68: 164-165.

DOI: $10.1183 / 09031936.00036408$

\title{
Predicting outcome of nasal surgery in patients with obstructive sleep apnoea
}

To the Editors:

In a recent randomised sham-surgery-controlled trial, KOUTSOURELAKIS et al. [1] demonstrated that nasal surgery for fixed nasal obstruction did not improve, or even worsened, sleep-disordered breathing in a majority of 27 patients with obstructive sleep apnoea (OSA) syndrome.

Based on previous studies showing that the oral breathing route predisposes to OSA [2], KOUTSOURELAKIS et al. [1] compared the proportion of nasal breathing during sleep in responders and nonresponders to nasal surgery.

This post hoc analysis revealed that four responders spent a low proportion of the pre-operative sleep study with nasal breathing, as assessed via nasal cannula/pressure transducer and oral thermistor measurements. KOUTSOURELAKIS et al. [1] concluded that analysis of the oral-nasal breathing route during sleep might help to identify favourable candidates for nasal surgery among OSA patients with fixed nasal obstruction. As KoUTSOURELAKIS et al. [1] pointed out, their technique for assessing impaired nasal breathing during sleep was not ideal since it could not quantify nasal ventilation, and conventional rhinomanometry was also unhelpful in predicting the success of nasal surgery.

It has previously been shown that nasal resistance (and nasal airflow) is highly variable over time, with changes in body position and during sleep [3]. The cyclical changes in sidepredominance of nasal airflow over time (the nasal cycles) additionally contribute to the high variability of nasal resistance, particularly in the presence of anatomical obstacles. It is, therefore, unsurprising that the outcome of nasal surgery cannot be reliably predicted from measurement of nasal resistance by rhinomanometry during wakefulness [4] or from qualitative oral-nasal flow recordings. An unobtrusive technique based on a bilateral nasal cannula/pressure transducer system that permits side-selective quantitative measurements of nasal airflow and conductance over the course of a night has recently been developed and validated $[3,5]$.

We suggest that future studies on the effects of nasal surgery or pharmacological treatment on sleep-disordered breathing should include continuous quantitative nocturnal nasal conductance measurements for the accurate assessment of a subject's nasal patency as a means of identifying potential responders to treatment.

\author{
M. Kohler* and K.E. Bloch ${ }^{\#}$ \\ *Oxford Centre for Respiratory Medicine, Churchill Hospital, \\ Oxford, UK, and "Pulmonary Division, University Hospital of \\ Zurich, Zurich, Switzerland.
}

\section{STATEMENT OF INTEREST}

None declared.

\section{REFERENCES}

1 Koutsourelakis I, Georgoulopoulos G, Perraki E, Vagiakis E, Roussos C, Zakynthinos SG. Randomised trial of nasal surgery for fixed nasal obstruction in obstructive sleep apnoea. Eur Respir J 2008; 31: 110-117.

2 Koutsourelakis I, Vagiakis E, Roussos C, Zakynthinos SG. Obstructive sleep apnoea and oral breathing in patients free of nasal obstruction. Eur Respir J 2006; 28: 1222-1228.

3 Kohler M, Thurnheer R, Bloch KE. Side-selective, unobtrusive monitoring of nasal airflow and conductance. J Appl Physiol 2006; 101: 1760-1765.

4 Kohler M, Bloch KE, Stradling JR. The role of the nose in the pathogenesis of obstructive sleep apnoea and snoring. Eur Respir J 2007; 30: 1208-1215.

5 Kohler M, Thurnheer R, Bloch KE. Non-invasive, sideselective nasal airflow monitoring. Physiol Meas 2005; 26: 69-82.

DOI: $10.1183 / 09031936.00012108$

\section{From the authors:}

We would like to thank M. Kohler and K.E. Bloch for their interest and comments about our recent paper [1] and we appreciate the opportunity to make some remarks in reply to their letter. 
Indeed, we have acknowledged that the unobtrusive instrumentation used in our study (nasal cannula/pressure transducer and oral thermistor) could not quantify ventilation [2]. Nonetheless, it is considered ideal for a qualitative characterisation of sleep epochs according to the predominant signal in either the nasal or the oral channel [3,4]. Undoubtedly, the "gold standard" for quantitative monitoring of nasal or oral ventilation is a cumbersome dual compartment face mask with attached pneumotachographs [5]. Additionally, the technique described by KOHLER et al. [6] that quantifies overnight nasal airflow and computes side-selective nasal resistance, albeit less obtrusive than the later, still requires similar instrumentation and a rather complex calibration procedure.

We have demonstrated that nasal surgery outcome could be predicted with high sensitivity and specificity by the qualitative nasal-oral flow recordings, whereas nasal resistance measured at the beginning of the sleep study did not have any predictive value. As KOHLER et al. [6] demonstrated, a single measurement does not properly reflect the highly variable values of nasal resistance during an entire night sleep. This point might be crucial for interpreting the findings of our study. Indeed, patients with fixed nasal obstruction who present predominant nasal breathing epochs during sleep may be those patients whose overnight nasal patency improves. These patients would represent the nonresponders of our study. On the contrary, patients with fixed nasal obstruction who present predominant oral or oronasal breathing epochs during sleep may be those patients whose overnight nasal patency remains unchanged or even worsens. These patients would represent the responders of our study because nasal surgery could succeed in normalising overnight nasal resistance. Nevertheless, this interesting link between the overnight variations of nasal resistance and the response to nasal surgery remains to be confirmed by future studies.

\section{Koutsourelakis and S. Zakynthinos}

Center of Sleep Disorders, Medical School of Athens University, Dept of Critical Care and Pulmonary Services, Evangelismos Hospital, Athens, Greece.

\section{STATEMENT OF INTEREST}

None declared.

\section{REFERENCES}

1 Koutsourelakis I, Georgoulopoulos G, Perraki E, Vagiakis E, Roussos C, Zakynthinos SG. Randomised trial of nasal surgery for fixed nasal obstruction in obstructive sleep apnoea. Eur Respir J 2008; 31: 110-117.

2 Koutsourelakis I, Vagiakis E, Roussos C, Zakynthinos S. Obstructive sleep apnoea and oral breathing in patients free of nasal obstruction. Eur Respir J 2006; 28: 1222-1228.

3 Sleep-related breathing disorders in adults, recommendations for syndrome definition and measurement techniques in clinical research. The Report of an American Academy of Sleep Medicine Task Force. Sleep 1999; 22: 667-689.

4 Montserrat J, Farré R, Ballester E, Felez M, Pastó M, Navajas D. Evaluation of nasal prongs for estimating nasal flow. Am J Respir Crit Care Med 1997; 155: 211-215.

5 Fitzpatrick MF, Driver HS, Chata N, Voduc N, Girard AM. Partitioning of inhaled ventilation between the nasal and oral routes during sleep in normal subjects. J Appl Physiol 2003; 94: 883-890.

6 Kohler M, Thurnheer R, Bloch KE. Side-selective, unobtrusive monitoring of nasal airflow and conductance. J Appl Physiol 2006; 101: 1760-1765.

DOI: 10.1183/09031936.00025808 